\title{
A comparison of brain phantom relative permittivity with CST simulation library and existing research
}

\author{
Kim Mey Chew, Norhudah Seman*, Rubita Sudirman and Ching Yee Yong \\ Faculty of Electrical Engineering, Universiti Teknologi Malaysia, 81310 Skudai, Johor, Malaysia
}

\begin{abstract}
The development of human-like brain phantom is important for data acquisition in microwave imaging. The characteristics of the phantom should be based on the real human body dielectric properties such as relative permittivity. The development of phantom includes the greymatter and whitematter regions, each with a relative permittivity of 38 and 28 respectively at $10 \mathrm{GHz}$ frequency. Results were compared with the value obtained from the standard library of Computer Simulation Technology (CST) simulation application and the existing research by Fernandez and Gabriel. Our experimental results show a positive outcome, in which the proposed mixture was adequate to represent real human brain for data acquisition.
\end{abstract}

Keywords: Relative permittivity, comparison, human-like brain phantom, CST simulation application

\section{Introduction}

Ultrasound, Magnetic Resonance Imaging (MRI), X-Ray Computed Tomography (CT), and Positive Emission Tomography (PET) scans are common imaging technologies used for detecting brain tumors. However, usage of these imaging tools may also cause adverse effects and inconveniences for the patient. Due to these limitations and disadvantages, microwave imaging has been proposed as a potential alternative. Tumor detection using microwave imaging relies on the difference in contrast between benign and malignant cells. The successful application of microwave imaging to breast tumor detection showcases its diagnostic potential for future detection of brain tumors [1].

Microwave imaging technique provides a well-defined view of the internal structure of an object by illuminating it with a low power electromagnetic wave at microwave frequencies. In mono-static microwave imaging system, a single ultra-wideband (UWB) antenna transmits a short pulse into the phantom. The backscattering parameters, $S_{11}$ are then received by the same antenna [2]. The collected data are then processed using signal and image processing techniques to form a $2 \mathrm{D}$ image for tumor size and location.

\footnotetext{
${ }^{*}$ Corresponding author: Norhudah Seman, Faculty of Electrical Engineering, Universiti Teknologi Malaysia, 81310 Skudai, Johor, Malaysia. Tel.: +607 5535265 ext.35265; Fax:+607 55 35252; E-mail: huda@fke.utm.my.
}

0959-2989/14/\$27.50 @ 2014 - IOS Press and the authors. 
In microwave imaging, relative permittivity is one of the dielectric properties that need to be considered for human brain phantom development [3]. In this study, the results of the developed phantom were validated against existing research results from Fernandez [4] and Gabriel [5], as well as the CST standard library.

\section{Literature review}

\subsection{Microwave imaging}

Microwave imaging is a process that involves scanning and 'seeing' an object's internal organ structure. The electromagnetic waveform with a frequency range between $300 \mathrm{MHz}$ and $30 \mathrm{GHz}$ is classified as microwave [6]. Jacobi and Larsen were the original scientists to conduct experiments extensively on the imaging of canine kidneys using microwave, non-ionizing radiation in the late 70s [7-9]. This was the pioneer experiment of microwave imaging in the biomedical field [10].

In microwave imaging, microwave functions as the transmitting wave. The wavelets used in microwave imaging and microwave ovens are different in terms of the power source. The microwave used for imaging consumes lower power source than the one used in an oven. A microwave oven uses mostly between 600 and 800 Watt of power on average, which is $125 \%$ higher than that used for microwave imaging (1 Watt) [11]. Therefore, microwave without heat emission is safe for medical imaging.

\subsection{Dielectric properties}

The dielectric properties of human tissues are essential information for researchers and scientists in the field of imaging, electromagnetic (EM), and biological systems. Each material has its unique electrical characteristic such as relative permittivity of dielectric properties [2].

Gabriel and colleagues [5] developed an application [12] that computes the dielectric properties of the tissues of the human body between $10 \mathrm{~Hz}$ and $100 \mathrm{GHz}$ frequencies using a parametric model and parameter values. It is capable $[13,14]$ of computing dielectric properties such as relative permittivity $\left(\varepsilon_{r}\right)$, electrical conductivity and a number of significantly derived quantities such as wavelength, loss tangent and skin depth penetration. For this study, a brain phantom was developed based on the dielectric properties of human tissues computed using this application. Eight main parts of

Table 1

Dielectric properties of human tissues from $1 \mathrm{GHz}$ to $10 \mathrm{GHz}$ frequencies [12]

\begin{tabular}{lll}
\hline Body tissues & Relative permittivity, $\varepsilon_{r}$ & Penetration $(\mathrm{m})$ \\
\hline Breast & $5.407-3.880$ & $0.235-0.014$ \\
Colon and rectum & $57.482-41.912$ & $0.036-0.003$ \\
Esophagus & $64.797-48.920$ & $0.035-0.003$ \\
Liver & $46.401-32.450$ & $0.041-0.003$ \\
Lung and bronchus & $51.102-37.951$ & $0.043-0.003$ \\
Prostate & $60.259-45.248$ & $0.033-0.003$ \\
Stomach & $64.797-48.920$ & $0.035-0.003$ \\
Uterine & $49.582-37.693$ & $0.038-0.003$ \\
Braingrey & $52.280-38.112$ & $0.039-0.003$ \\
Brainwhite & $38.570-28.395$ & $0.054-0.004$ \\
\hline
\end{tabular}


the human body have a high incidence of cancer [15] and their dielectric properties are shown in Table 1.

\section{Methodology}

The dielectric properties were measured using HP 8720B Vector Network Analyzer (VNA) and HP 85070B open-ended coaxial sensor. The measurement was performed by submerging the probe into the liquid or touching the flat face of a solid material. The material should have sufficient thickness to establish an 'infinite' status with the probe for transmitting and receiving the measured data [2].

\subsection{Calibration and verification using water}

In this study, the calibration and verification processes were conducted according to the dielectric properties of water. Experimentally measured dielectric properties of water were verified with the material properties library in the simulation application, Computer Simulation Technology (CST) and the results from Fernandez [4]. Calibration and verification processes are important for data acquisition process in order to avoid bias and error.

\subsubsection{Mixture elements}

In order to develop the proposed phantom, the selected elements were selected according to the soft characteristic of the brain, like tofu or soft gelatine. Figure 1 shows the proposed mixture, which consisted of gelatine, distilled water and edible sugar by mixing them proportionately. For the purpose of developing a human-like brain phantom, the proposed mixture has to meet the relative permittivity, $\varepsilon_{r}$ values for the greymatter and whitematter regions. The proposed mixtures were as follows:

- Mixture $1=100 \mathrm{ml}$ water $+10 \mathrm{~g}$ gelatine $+25 \mathrm{~g}$ sugar

- Mixture $2=100 \mathrm{ml}$ water $+10 \mathrm{~g}$ gelatine $+26 \mathrm{~g}$ sugar

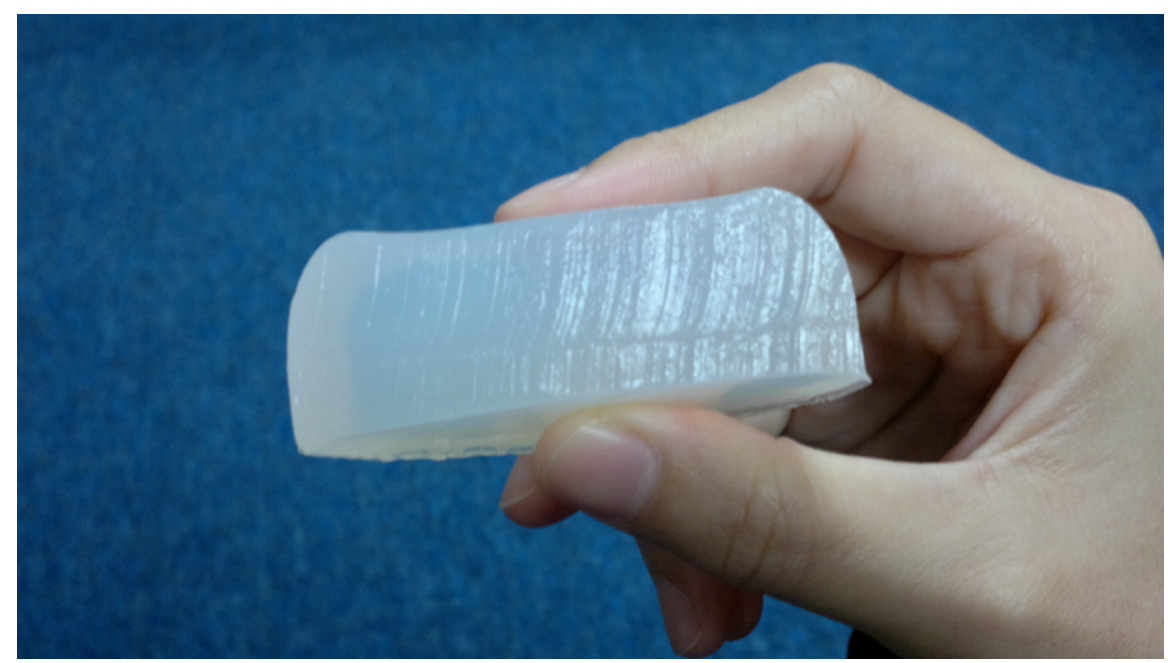

Fig. 1. Experimental mixture/model consisted of gelatine, distilled water and edible sugar. 
- Mixture $3=100 \mathrm{ml}$ water $+10 \mathrm{~g}$ gelatine $+27 \mathrm{~g}$ sugar

- Mixture $4=100 \mathrm{ml}$ water $+10 \mathrm{~g}$ gelatine $+28 \mathrm{~g}$ sugar

- Mixture $5=100 \mathrm{ml}$ water $+10 \mathrm{~g}$ gelatine $+29 \mathrm{~g}$ sugar

Small beakers were used to place these samples for permittivity probing.

\section{Results}

Although the study is in its initial stage, this pilot test was performed as a basic test and analysis on the gathered data. The key objective of the study was to formulate a mixture that can represent the human brain for microwave imaging. The experiment results were compared to the CST material library and existing research.

A comparison of relative permittivity, $\varepsilon_{r}$ was done by covering the frequency range between 1 and 10 GHz. Table 2 shows the relative permittivity of water collected from the experiment, Computer Simulation Technology (CST) and existing research (Fernandez [4]), while Table 3 demonstrates the relative permittivity of the brain (greymatter and whitematter) obtained from Computer Simulation Technology (CST), existing research (Gabriel [5]) and the experiment. Figure 2 shows the plot for relative permittivity based on Table 2, while Figure 3 shows the plot for relative permittivity based on Table 3.

Table 2

Relative permittivity of water from $1-10 \mathrm{GHz}$

\begin{tabular}{ll}
\hline Source & Relative Permittivity, $\varepsilon_{r}$ \\
\hline CST & $\varepsilon_{r} \sim 83-78$ \\
Fernandez & $\varepsilon_{r} \sim 77-72$ \\
Experiment & $\varepsilon_{r} \sim 77-68$ \\
\hline
\end{tabular}

Table 3

Relative permittivity for brain for $1-10 \mathrm{GHz}$

\begin{tabular}{lll}
\hline \multirow{2}{*}{ Source } & \multicolumn{2}{l}{ Relative Permittivity, $\varepsilon_{r}$} \\
\cline { 2 - 3 } Greymatter & Whitematter \\
\hline CST & $\varepsilon_{r} \sim 189-42$ & - \\
\hline Gabriel & $\varepsilon_{r} \sim 52-38$ & $\varepsilon_{r} \sim 38-28$ \\
\hline Experiment & & \\
\hline Mixture 1 & $\varepsilon_{r} \sim 54.43-29.75$ & - \\
\hline Mixture 2 & $\varepsilon_{r} \sim 53.97-29.38$ & - \\
\hline Mixture 3 & $\varepsilon_{r} \sim 53.78-29.05$ & - \\
\hline Mixture 4 & $\varepsilon_{r} \sim 53.49-28.75$ & - \\
\hline Mixture 5 & $\varepsilon_{r} \sim 53.47-27.92$ & - \\
\hline
\end{tabular}




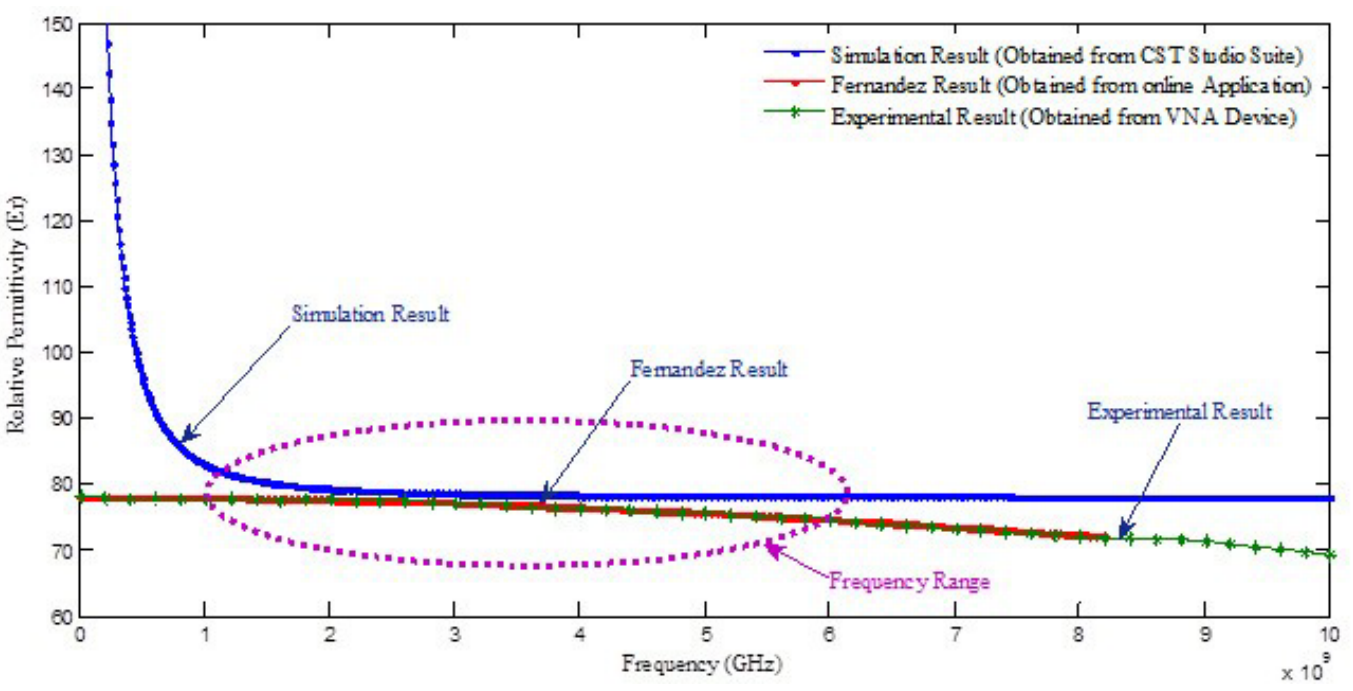

Fig. 2. Relative permittivity of water from simulation, Fernandez [4] and experimental result.

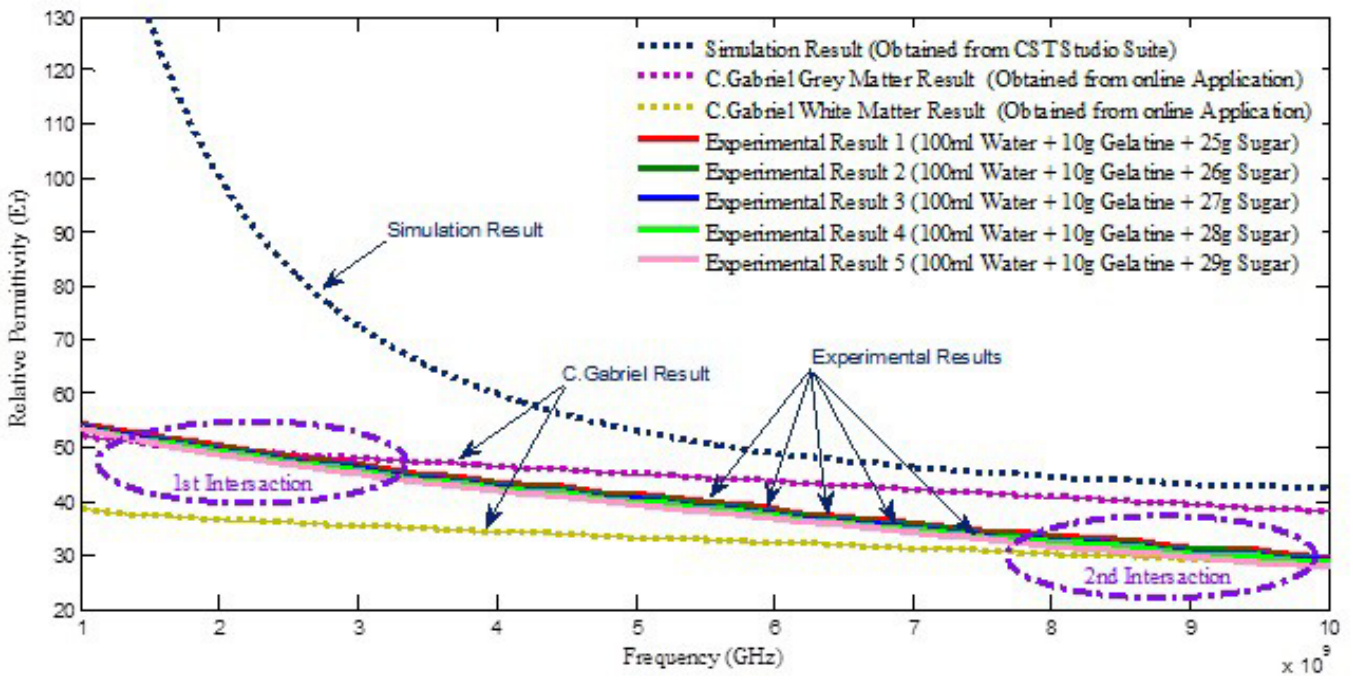

Fig. 3. Relative permittivity of brain from simulation, Gabriel [5] and experimental result.

\subsection{Water}

In Figure 2, CST simulation showed that water achieved a steady state between the frequencies 2 and $10 \mathrm{GHz}$ with relative permittivity $\varepsilon_{r} \sim 79-78$. According to the plots, the experimental result of theproposed model matched the results of Fernandez [4]. The plots for both Fernandez [4] and the proposed xperimental model had almost similar relative permittivity, $\varepsilon_{r}$ values compared to the simulation result. The result shows that $100 \mathrm{ml}$ of water was appropriate and accurate as solvent for the mixture. 


\subsection{The greymatter and whitematter in human brain}

The relative permittivity, $\varepsilon_{r}$ values collected from the CST simulation application library for the greymatter is shown Figure 3. Based on the graph, there was a steep decline from $1 \mathrm{GHz}$ to $6 \mathrm{GHz}$. The decline was followed by steady-state alleviation at frequencies 6 to $10 \mathrm{GHz}$ with relative permittivity, $\varepsilon_{r} \sim 53-42$.

According to Gabriel [5], the plots for greymatter and whitematter were recorded an alleviate decrement

from 1 to $10 \mathrm{GHz}$. The experimental and simulated greymatter plots were similar to each other between 6 and $10 \mathrm{GHz}$.

Previous studies $[3,16]$ reported that when sugar concentration increased, relative permittivity, $\varepsilon_{r}$ decreased. The experimental plot contained two points that interacted with Gabriel's [4] greymatter and whitematter plots, whereby the first interaction point was for the greymatter plot, located between 1 and $3 \mathrm{GHz}$, while the second interaction point was for the whitematter plot, located between 8 and 10 $\mathrm{GHz}$.

\section{Discussion}

The experimental, CST simulation and Fernandez's [4] relative permittivity, $\varepsilon_{\mathrm{r}}$ plots for water are shown in Figure 2. Results demonstrated that the experimental result was similar to CST simulation and Fernandez's result [4].

Based on Figure 3, the plots for the proposed greymatter and whitematter mixture were close with Gabriel's result [5] for frequencies between 1 and $3 \mathrm{GHz}$ and 8 and $10 \mathrm{GHz}$, respectively. The relative ermittivity, $\varepsilon_{\mathrm{r}}$ differed by \pm 8 , but the variation was considered acceptable. The intersecting points uggest that the elements of the proposed mixture were sufficient for the development of human brain hantom. In order to form the greymatter layer, the sugar concentration has to be adjusted. The decrement of sugar concentration allows the increment of the relative permittivity, $\varepsilon_{\mathrm{r}}$ value and viceversa. Hence, the decrement of sugar amount has to be considered to achieve the targeted relative permittivity value for the greymatter region development, while the increment is considered to achieve the targeted relative permittivity value for the whitematter region development.

In all cases, there were significant differences between CST simulation and experimental results at the low frequency range due to the different algorithms and calibration methods used in the simulation.

Furthermore, sugar has a role in stabilizing the model's permittivity, $\varepsilon_{\mathrm{r}}$ values. Increasing sugar concentration leads to the decreased permittivity of a sample and vice-versa. Hence, future experiments should focus on building a real-like brain phantom, consisting of greymatter, whitematter and tumor.

\section{Conclusion}

The performance of the proposed mixture was compared with the CST simulation result, as well as the results of Fernandez [4] and Gabriel [5]. The elements of the mixture (gelatine, distilled water and edible sugar) were suitable for developing a human brain phantom. Future work is needed to develop the greymatter and whitematter layers with more accurate relative permittivity value. 


\section{Acknowledgement}

The authors would like to thank Universiti Teknologi Malaysia and the Ministry of Education for supporting and funding this research under the Fundamental Research Grant Scheme (4F206) and MyPhd Scholarship scheme.

\section{References}

[1] W.C. Khor, Microwave imaging for breast cancer detection, Ph.D. Dissertation, University of Queensland, 2010.

[2] Agilent basics of measuring the dielectric properties of materials, Agilent Literature Number 5989-2589EN, Agilent, 2006.

[3] K.M. Chew, R. Sudirman, N. Seman and C.Y. Yong, Human brain phantom modeling based on relative permittivity dielectric properties, 2012 International Conference on Biomedical Engineering and Biotechnology (ICBEB) 239 (2012), 817-820.

[4] D.P. Fernandez, A.R.H. Goodwin, E.W. Lemmon, J.L. Sengers and R.C. Williams, A formulation for the static permittivity of water and steam at temperatures from $238 \mathrm{k}$ to $873 \mathrm{k}$ at pressures up to $1200 \mathrm{mpa}$, including derivatives and debye-huckel coefficients, Journal of Physical and Chemical Reference Data 26 (1997), 1125-1166.

[5] S. Gabriel, R.W. Lau and C. Gabriel, The dielectric properties of biological tissues: iii. Parametric models for the dielectric spectrum of tissues, Physics in Medicine and Biology 41 (1996), 2271.

[6] E.C. Fear, P.M. Meaney and M.A. Stuchly, Microwaves for breast cancer detection? Potentials, IEEE 22 (2003), 12-18.

[7] L.E. Larsen, Medical applications of microwave imaging, in: The 1980 International Microwave Symposium, IEEE Press, New York, 1986, pp. 1-229.

[8] L.E. Larsen and J.H. Jacobi, Microwave Interrogation of dielectric targets, part I: By scattering parameters, Medical Physics 5 (1978), 500-508.

[9] J.H. Jacobi and L.E. Larsen, Microwave interrogation of dielectric targets, part II: By microwave time delay spectroscopy, Medical Physics 5 (1978), 509-513.

[10] J.H. Jacobi, L.E. Larsen and C.T. Hast, Water-immersed microwave antennas and their application to microwave interrogation of biological targets, IEEE Transactions on Microwave Theory and Techniques 27 (1979), 70-78.

[11] A. Kamerman and N. Erkocevic, Microwave oven interference on wireless LANs operating in the $2.4 \mathrm{GHz}$ ISM band, The 8th IEEE International Symposium on Personal, Indoor and Mobile Radio Communications (PIMRC) 3 (1997), $1221-1227$.

[12] Dielectric properties of body tissues in the frequency range $10 \mathrm{~Hz}$ to $100 \mathrm{GHz}$, Available at: http://niremf.ifac.cnr.it/tissprop/, June 10th, 2014.

[13] K.M. Chew, R. Sudirman, N. Seman and C.Y. Yong, Reflection coefficient detection of simulation models for microwave imaging simulation system, Bio-Medical Materials and Engineering 24 (2014), 199-207.

[14] T. Yoshikawa, H. Ohgushi, T. Uemura, H. Nakajima, K. Ichijima, S. Tamai and T. Tateisi, Human marrow cells-derived cultured bone in porous ceramics, Bio-Medical Materials and Engineering 8 (1998), 311-320.

[15] F. Pfizer, The Burden of Cancer in Asia 2008, Pfizer Medical Division, United States of America, 2008, pp. 1-83

[16] K.M. Chew, R. Sudirman, N. Seman and C.Y. Yong, Human brain phantom modeling: Concentration and temperature effects on relative permittivity, Advanced Materials Research 646 (2013), 191-196. 